\title{
Linear Breit-Wheeler pair production by high-energy bremsstrahlung photons colliding with an intense $x$-ray laser pulse
}

\author{
A. Golub, ${ }^{1, *}$ S. Villalba-Chávez, ${ }^{1, \dagger}$ H. Ruhl@ ${ }^{2, \ddagger}$ and C. Müller ${ }^{1,}$ \\ ${ }^{1}$ Institut für Theoretische Physik I, Heinrich-Heine-Universität Düsseldorf, \\ Universitätsstraße 1, 40225 Düsseldorf, Germany \\ ${ }^{2}$ Arnold Sommerfeld Center, LMU Munich, Theresienstraße 37, 80333 München, Germany
}

(Received 8 October 2020; accepted 18 December 2020; published 12 January 2021)

\begin{abstract}
A possible setup for the experimental verification of linear Breit-Wheeler pair creation of electrons and positrons in photon-photon collisions is studied theoretically. It combines highly energetic bremsstrahlung photons, which are assumed to be generated by an incident beam of $\mathrm{GeV}$ electrons penetrating through a high- $Z$ target, with $\mathrm{keV}$ photons from an $\mathrm{x}$-ray laser field, which is described as a focused Gaussian pulse. We discuss the dependencies of the pair yields on the incident electron energy, target thickness, laser parameters, and collision geometry. It is shown that, for suitable conditions that are nowadays in reach at $\mathrm{x}$-ray laser facilities, the resulting number of created particles seems to be well accessible for enabling the first experimental observation of the linear Breit-Wheeler process $\gamma \gamma \rightarrow e^{+} e^{-}$.
\end{abstract}

DOI: 10.1103/PhysRevD.103.016009

\section{INTRODUCTION}

Production of electrons and positrons in a collision of photons represents one of the most intriguing predictions of quantum electrodynamics (QED) as it implies that matter can be created from light. Although the underlying fundamental process $\gamma \gamma \rightarrow e^{+} e^{-}$was described theoretically by Breit and Wheeler long ago [1], experimental validation of electron-positron pair production from light has so far been accomplished solely in the nonlinear multiphoton regime as part of a two-step process [2,3]: first, the interaction of a $46.6 \mathrm{GeV}$ electron beam with an optical terawatt laser pulse at the Stanford Linear Accelerator Center (SLAC) provided backscattered photons of multi-GeV energy which, subsequently, created electron-positron pairs upon collision with several $\mathrm{eV}$ laser photons (nonlinear Breit-Wheeler process [4]). Following energy-momentum conservation with inclusion of field-dressing effects, at least five laser photons were needed in the second reaction step to overcome the energy threshold [5,6], and a total of about 100 positrons was detected during the experiment.

\footnotetext{
*Alina.Golub@uni-duesseldorf.de †selym@tp1.uni-duesseldorf.de

thartmut.ruhl@physik.uni-muenchen.de

§c.mueller@tp1.uni-duesseldorf.de
}

Published by the American Physical Society under the terms of the Creative Commons Attribution 4.0 International license. Further distribution of this work must maintain attribution to the author(s) and the published article's title, journal citation, and DOI. Funded by SCOAP.
The successful SLAC experiment, in combination with the ongoing development of high-intensity laser technology, has triggered a substantial theoretical interest in laser-induced pair production and, particularly, in the Breit-Wheeler process $[7,8]$. On the one side, there is a research focus on the highly nonlinear regime of this process [9-16], where its rate exhibits an exponential nonperturbative field dependence resembling the famous Schwinger rate. Besides, the influence of the precise shape of the laser field has been examined in detail (see $[11,17-24]$ and references therein). On the other side, also the linear version of the process [25], as originally studied by Breit and Wheeler, where an electron-positron pair is produced in a collision of two photons, has been under active scrutiny by theoreticians [26-32]. While being known to play a crucial role in various astrophysical contexts, such as $\gamma$-ray bursts, black hole dynamics, and active galactic nuclei $[33,34]$, this elementary QED effect has not been validated experimentally in the laboratory yet.

The difficulty for observing the linear Breit-Wheeler effect manifests itself when the energy-momentum conservation is considered

$$
k+k^{\prime}=p_{+}+p_{-}
$$

where $k$ and $k^{\prime}$ stand for the photons wave vectors and $p_{ \pm}$ denote the electron and positron four momenta, correspondingly. It leads to the threshold relation $\omega \omega^{\prime} \geq \frac{2 m^{2}}{1-\cos (\phi)}$ between the photon energies $\omega$ and $\omega^{\prime}$, the particles' rest mass $m$, and the angle $\phi$ between the photon propagation directions (see Fig. 1). Accordingly, in the center-of-mass 


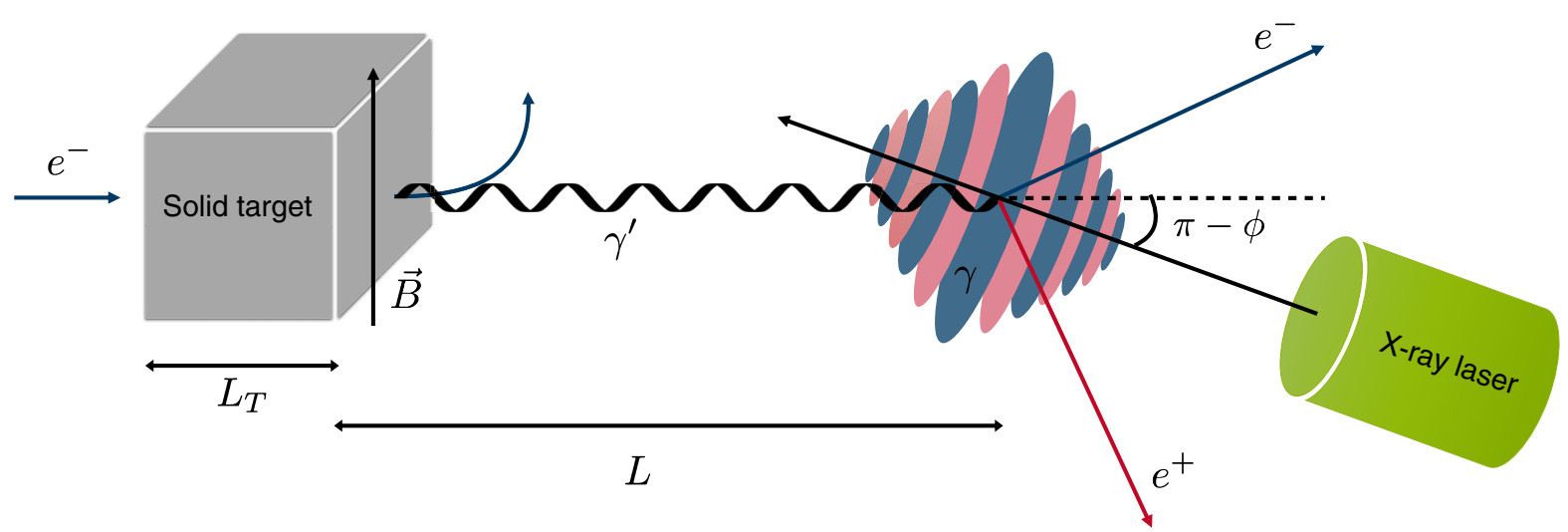

FIG. 1. Setup of the proposed experiment for electron $\left(e^{-}\right)$and positron $\left(e^{+}\right)$creation. Here, the GeV and keV photons involved in the reaction are provided by bremsstrahlung and $\mathrm{x}$-ray laser radiation, respectively.

frame, the photon energies need to be of the $\mathrm{MeV}$ order- $\mathrm{a}$ scenario that is challenging to achieve nowadays with sufficiently high beam intensities [35]. Throughout this paper we work in Lorentz-Heaviside units where $c=\hbar=$ $\epsilon_{0}=1$ and use the metric with signature $\operatorname{diag}\left(g^{\mu \nu}\right)=$ $(1,-1,-1,-1)$.

In recent years, various theoretical proposals for detection of linear Breit-Wheeler pair production were put forward. The first designs relied on photon-photon colliders, where one source was provided by thermal hohlraum radiation, whereas the partner photon was either described as a plane electromagnetic wave [26] or supposed to result from bremsstrahlung inside a high- $Z$ target [27]. These proposals were followed by symmetric setups incorporating photons stemming from two equal origins, such as Compton gamma sources [28], laser pulses interacting with thin aluminium targets or dense, short gas jets [29] or multiPW laser beams penetrating through narrow tube targets [30,31]. Finally, the possibility of detecting an analog of the Breit-Wheeler process in band gapped graphene layers at a much lower energy scale has recently been discussed [32].

In the current paper, we propose an alternative approach to detect the linear Breit-Wheeler process, which seems to be feasible in the nearest future, as the involved techniques are well established nowadays. The setup under consideration relies on few-GeV bremsstrahlung photons interacting with $\mathrm{keV}$ photons from an $\mathrm{x}$-ray free-electron laser (XFEL) beam, this way providing the energy needed for the process to take place. The bremsstrahlung is assumed to be generated by a $\mathrm{GeV}$ electron beam penetrating through a high- $Z$ solid target; as a possible source of the highly relativistic incident electrons we propose laser wakefield acceleration (LWFA), which has proven itself to be suitable for the production of narrowly collimated intense electron beams $[36,37]$. We point out that the required ingredients for our scheme are in principle available at the HiBEF facility [38] at DESY (Hamburg, Germany), where a shortpulse 300-terawatt optical laser is operated in conjunction with the European XFEL.
It is important to note that Bethe-Heitler pair production in the high- $Z$ target represents a competing channel for positron creation, as the bremsstrahlung photons may decay into pairs in the atomic Coulomb fields of the solid $[39,40]$. Therefore, in order to ensure the interaction volume being free from disturbance caused by electrons from the incident beam or potentially created Bethe-Heitler pairs, a static magnetic field needs to be introduced sidetracking the charged particles. Other QED processes are neglectable in the considered parameter range. A schematic diagram of the proposed setup is shown in Fig. 1.

The initial idea to probe Breit-Wheeler pair creation by bremsstrahlung-laser interaction goes back to Reiss [41]. Currently, similar schemes are investigated in further detail $[13,14,42]$. Involving high-intensity optical laser pulses, they aim at the detection of the nonlinear Breit-Wheeler process in the nonperturbative regime. Predictions for the expected pair yields were made $[13,14]$ and the possibility to exploit the process for experimentally determining the critical Schwinger field of QED was discussed [14]. The latter study considered, in particular, the parameter range envisaged by the LUXE experiment at DESY, which aims at an implementation of an optical laser-bremsstrahlung collider for detection of nonlinear Breit-Wheeler pair creation [42]. In contrast to these developments, our present proposal relies on an intense $\mathrm{x}$-ray laser beam to probe the linear Breit-Wheeler process $\gamma \gamma \rightarrow e^{+} e^{-}$. With respect to Refs. [13,14,42], our study has, thus, a complementary character, targeting on the opposite asymptotic regime of the process.

Our paper is structured in the following way: after the introduction, we present in Sec. II the theoretical framework for describing the linear Breit-Wheeler process in a collision of a highly energetic bremsstrahlung photon with an $\mathrm{x}$-ray laser photon. The laser light is modeled as a linearly polarized, monochromatic Gaussian pulse and the spectrum of bremsstrahlung radiation is taken into account. Section III illustrates our results and provides estimations for the number of created positrons that can be detected in 
dependence on the various setup parameters. In the last section a conclusion is provided and, finally, some technical details are given in an Appendix.

\section{THEORETICAL DESCRIPTION}

This section is devoted to a theoretical perturbative development of linear electron-positron pair creation occurring when bremsstrahlung photons impinge on photons coming from a laser field. In contrast to the well-established consideration, where both photons are treated as quantized modes, we model the laser photons as a classical Gaussian pulse. In this context the Volkov-states approach, which is customarily pursued in strong-field QED [4], is not applicable as we are dealing with a focused field. Hence, we shall first formulate a theoretical framework whichstarting from a fully quantum picture-allows us to go smoothly over to a description of the interaction between a classical laser field and an arbitrary quantized photon mode (Sec. II A). This approach will afterwards enable to incorporate the field focusing (Sec. II B) as well as the spectrum of bremsstrahlung photons (Sec. IIC) in a natural way.

\section{A. Pair production in a laser field}

In the parameter range of interest-where the maximum amplitude $\mathcal{E}_{0}$ of the laser electric field satisfies $|e| \mathcal{E}_{0} \ll m \omega$ - the linear Breit-Wheeler process is described by the second-order scattering matrix element $S_{f i}$. It is usually expressed fully quantum mechanically with the two initial photons and the created electronpositron pair treated as number states [43,44]. In order to include a laser field, one of the photon number states can be replaced by a coherent state $\left|\alpha_{k}\right\rangle$ in the mode $k$ while keeping the description of the remaining constituents as $\left|k^{\prime}, \epsilon^{\prime}\right\rangle$ and $\left|p_{+}, \sigma_{+} ; p_{-}, \sigma_{-}\right\rangle$, correspondingly [45]. Here, $k^{\prime}$ is the wave four vector of the involved bremsstrahlung photon with polarization $\epsilon^{\prime}$, whereas $p_{ \pm}, \sigma_{ \pm}$stand for the electron and positron four-momenta and spin states. Accordingly, we obtain

$$
\begin{aligned}
S_{f i} & =\left\langle p_{+}, \sigma_{+} ; p_{-}, \sigma_{-} ; \alpha_{k}|\hat{S}[\hat{\psi}, \hat{\bar{\psi}}, \hat{a}]| k^{\prime}, \epsilon^{\prime} ; \alpha_{k}\right\rangle \\
& =\left\langle p_{+}, \sigma_{+} ; p_{-}, \sigma_{-}\left|\hat{S}\left[\hat{\psi}, \hat{\bar{\psi}}, \hat{a}+a_{\mathrm{ext}}\right]\right| k^{\prime}, \epsilon^{\prime}\right\rangle,
\end{aligned}
$$

with the QED scattering operator $\hat{S}[\hat{\psi}, \hat{\bar{\psi}}, \hat{a}]$ of the second order in the fermion $\hat{\psi}$ and gauge $\hat{a}$ field operators

$$
\begin{aligned}
& \hat{S}[\hat{\psi}, \hat{\bar{\psi}}, \hat{a}] \\
& \quad=\frac{(i e)^{2}}{2 !} \int d^{4} x d^{4} y \hat{\mathcal{T}}[\hat{\bar{\psi}}(x) \hat{\phi}(x) \hat{\psi}(x) \hat{\bar{\psi}}(y) \hat{\phi}(y) \hat{\psi}(y)],
\end{aligned}
$$

where we have introduced the Feynman notation $\hat{\phi}=\gamma^{\mu} a_{\mu}$ with Dirac gamma matrices $\gamma^{\mu}, \hat{\bar{\psi}}=\hat{\psi} \gamma \gamma^{0}$ and $\hat{\mathcal{T}}$ stands for the time-ordering operator. In the second line of Eq. (2) the results obtained in [46] were used. This procedure allows us to evaluate the consequence of the coherent state by adding to the photon field operator $\hat{a}^{\mu}(x)$ the classical electromagnetic field potential $a_{\mathrm{ext}}^{\mu}(x)=\epsilon^{\mu} a_{0}(x, k)$ with polarization $\epsilon^{\mu}\left(\epsilon^{\mu} k_{\mu}=0\right)$, which will be chosen in the radiation gauge. The quantization of the former has been carried out within the Gupta-Bleuler formalism. Based on the expression in the second line of Eq. (2) we can calculate the BreitWheeler process for a classical laser field of arbitrary shape, since the latter may be written as a linear superposition of wave modes. Accordingly, $a_{0}(x, k)$ shall represent the vector potential amplitude of a focused laser field with central wave four-vector $k^{\mu}$.

The rate per volume of the process is obtained as

$$
R_{\epsilon, \epsilon^{\prime}}^{\sigma_{ \pm}}\left(k, k^{\prime}\right)=\int \frac{V d^{3} p_{+}}{(2 \pi)^{3}} \frac{V d^{3} p_{-}}{(2 \pi)^{3}} \frac{\left|S_{f i}\right|^{2}}{T V},
$$

where the scattering matrix element is being integrated over the phase space of created electron and positron, while divided by the interaction time $T$ and volume $V$. In our context, after averaging over the polarization of the quantized photon as well as summation over the fermions spins, the rate per volume reads

$$
R\left(k, k^{\prime}\right)=\frac{1}{T V} \int \frac{d^{4} \tilde{k}}{(2 \pi)^{4}}\left|\tilde{a}_{0}(\tilde{k}, k)\right|^{2} \frac{R_{B W}\left(\tilde{k} k^{\prime}\right)}{N_{\gamma}^{2}},
$$

with the Fourier transform of the vector potential amplitude

$$
\tilde{a}_{0}(\tilde{k}, k)=\int d^{4} x \mathrm{e}^{i \tilde{k} x} a_{0}(x, k) .
$$

Moreover, the quantity $R_{B W}\left(\tilde{k} k^{\prime}\right) / N_{\gamma}^{2}$ stands for the wellknown rate of Breit-Wheeler pair creation obtained as a result of the collision of two gamma quanta

$$
\begin{aligned}
R_{B W}\left(\tilde{k} k^{\prime}\right)= & \frac{\alpha e^{2} N_{\gamma}^{2}}{\omega^{\prime} V_{\gamma}} u(s), \\
u(s)= & {\left[\frac{-s \sqrt{s^{2}-1}\left(1+s^{2}\right)}{s^{4}}\right.} \\
& \left.+\frac{\left(-1+2\left(s^{2}+s^{4}\right)\right) \ln \left(s+\sqrt{s^{2}-1}\right)}{s^{4}}\right],
\end{aligned}
$$

where $N_{\gamma}$ refers to the normalization constant of the quantized field. In this formula, $\alpha \approx 1 / 137$ is the finestructure constant, $V_{\gamma}$ is the quantization volume, and $s^{2}=$ $k k^{\prime} / 2 m^{2}$ is the normalized Mandelstam variable [4,43].

\section{B. Pair production in the field of a Gaussian pulse}

In this section we model the laser field by incorporating the vector potential of a linearly polarized Gaussian pulse in paraxial approximation propagating in the $z$ direction for 
$a_{\mathrm{ext}}^{\mu}$. As can be seen in Eq. (5), for proceeding further, we require the absolute value squared of the Fourier transformed amplitude $a_{0}(x, k)$, which is given in the Appendix. When considering Eq. (A5) the rate per volume in cylindrical coordinates reads

$$
\begin{aligned}
R\left(k, k^{\prime}\right)= & \frac{\mathcal{E}_{0}^{2} N_{\gamma}^{2}}{32} \frac{(\tau / 2)^{2} w_{0}^{4}}{T A} \int_{0}^{2 \pi} d \phi_{\tilde{k}} \int_{-\infty}^{\infty} d \tilde{k}_{z} \\
& \times \int_{0}^{\left|\tilde{k}_{0}\right|} d \tilde{k}_{\perp} \tilde{k}_{\perp} \mathrm{e}^{-\frac{\tilde{k}^{2} w_{0}^{2}}{2}} \int_{-\infty}^{\infty} \frac{d \tilde{k}_{0}}{\tilde{k}_{0}^{2}} \mathrm{e}^{-\frac{(\tau / 2)^{2}}{2}\left(\omega-\tilde{k}_{0}\right)^{2}} \\
& \times \delta\left(\tilde{k}_{z}-\tilde{k}_{0}+\frac{\tilde{k}_{\perp}^{2}}{2 \omega}\right) R_{B W}\left(\tilde{k} k^{\prime}\right) .
\end{aligned}
$$

Here, $A$ denotes the interaction area resulting from the interaction volume divided by a length factor, which stems from squaring the Dirac $\delta$ function. Next, we perform the integration over $\tilde{k}_{z}$ by exploiting the latter one and, afterwards, integrate over $\tilde{k}_{0}$ by evaluating all components except the exponential function at $\tilde{k}_{0}=\omega$ as it provides the biggest contribution to the integral. In that case, the argument of $R_{B W}$ can be approached by $\tilde{k} k^{\prime} \approx$ $\omega \omega^{\prime}\left(1-\cos \left(\phi_{\tilde{k}}\right) \sqrt{1+\frac{\tilde{k}_{\perp}^{4}}{4 \omega^{4}}}\right)$ and when substituting $v=$ $\frac{\tilde{k}_{\perp} w_{0}}{\sqrt{2}}$ we obtain

$$
\begin{aligned}
R\left(k, k^{\prime}\right) \approx & \frac{\mathcal{E}_{0}^{2} N_{\gamma}^{2}}{4 \omega^{2}} \frac{(\tau / 2)}{T A} \sqrt{\frac{\pi}{2}} \frac{w_{0}^{2}}{2} \int_{0}^{\frac{\omega w_{0}}{2}} d v v \mathrm{e}^{-v^{2}} \\
& \times \int_{0}^{2 \pi} d \phi_{\tilde{k}} R_{B W}\left[\omega \omega^{\prime}\left(1-\cos (\phi) \sqrt{1+\frac{v^{4}}{w_{0}^{4} \omega^{4}}}\right)\right] .
\end{aligned}
$$

The upper bound of integration in the radial component is restricted to $\frac{\omega w_{0}}{2}$ as $\tilde{k}_{\perp}$ is bounded to lie within the interval $[0, \omega]$. Moreover, we treat the collision angle $\phi$ between laser and quantized photon as an external parameter, which is predefined in our setup (see Fig. 1). Its value will be chosen in a way to allow for a practicable geometry of the experimental setup, which avoids damaging technical devices with the intense laser beam.

When taking into account the paraxial approximation $\left(\tilde{k}_{\perp} \ll \omega\right)$, the square root in $R_{B W}$ can be approached by 1 and, consequently, the rate of the process becomes

$R\left(\omega, \omega^{\prime}, \cos (\phi)\right) \approx \frac{a_{0}^{2}}{4 N_{\gamma}^{2}}\left(1-\mathrm{e}^{-\frac{\omega_{0}^{2} \omega^{2}}{4}}\right) R_{B W}\left[\omega \omega^{\prime}(1-\cos (\phi))\right]$.

Here, we have used the relations $T A=\frac{\tau}{2} \sqrt{\frac{\pi}{2}} \frac{\pi \omega_{0}^{2}}{2}$ as the laser pulse energy of the considered field reads $E_{L}=\frac{\mathcal{E}_{0}^{2}}{2} \frac{\tau}{2} \sqrt{\frac{\pi}{2}} \frac{\pi w_{0}^{2}}{2}$ [47]. Additionally, we denote $a_{0}=\mathcal{E}_{0} / \omega$.

\section{Bremsstrahlung photons}

To obtain the pair creation rate resulting from a collision of bremsstrahlung photons with a Gaussian laser pulse we integrate the rate, as given in Eq. (9), weighted by the distribution function of the bremsstrahlung photons $W\left(k^{\prime}\right)$ with respect to the photon momentum $k^{\prime}$ :

$$
R_{\gamma}(k)=\int \frac{d^{3} k^{\prime}}{(2 \pi)^{3}} W\left(k^{\prime}\right) R\left(k, k^{\prime}\right) .
$$

Since the incoming electrons employed in the proposed setup are highly relativistic, the generated bremsstrahlung photons will be emitted preferably in the direction of the incident electron propagation. More precisely, the angle of photon spreading can be approximated by $\theta \approx \frac{m \ln \left(E_{0} / m\right)}{E_{0}}$ [48], where $E_{0}$ stands for the initial kinetic energy of the incoming electrons. Hence, for $E_{0}$ of the order of $\mathrm{GeV}$ we will have angles in the mrad range. Considering this fact allows us to approximate the bremsstrahlung distribution function in spherical coordinates by

$W\left(k^{\prime}\right) \approx \frac{(2 \pi)^{3} I_{\gamma}(f, \ell)}{\omega^{\prime 2} \sin \left(\theta_{k^{\prime}}\right) E_{0}} \Theta\left(E_{0}-\omega^{\prime}\right) \delta\left(\theta_{k^{\prime}}-\phi\right) \delta\left(\phi_{k^{\prime}}\right)$

with the photon energy spectrum derived within the complete screening approximation [49]

$$
I_{\gamma}(f, \ell) \approx \frac{(1-f)^{\frac{4}{3} \ell}-\mathrm{e}^{-\frac{7}{9} \ell}}{f\left(\frac{7}{9}+\frac{4}{3} \ln (1-f)\right)},
$$

and $\phi$ being the angle between the directions of propagation of laser photons and bremsstrahlung electrons as both beams lie in one plane, whereas the laser beam propagates in the $z$ direction and its focal point is set to define the origin [see Eq. (A1)]. Moreover, the distribution function depends on the normalized target thickness $\ell=L_{T} / L_{\text {rad }}$ with $L_{\text {rad }}$ being the radiation length of the target material and $f=\omega^{\prime} / E_{0}$ stands for the normalized photon energy. Notice that the formula above provides a good estimation for the photon energy distribution in the ranges $0.5 \lesssim \ell \lesssim 2$ and $f \gtrsim 0.2=f_{\min }$ (see [49]).

Hence, when taking into account the assumptions listed above Eq. (10), the rate per volume of the process reads

$R_{\gamma}(\omega, \cos (\phi))=\int_{0}^{1} d f R\left(\omega, \omega^{\prime}, \cos (\phi)\right) I_{\gamma}(f, \ell)$.

This equation constitutes the basis for our numerical results which are presented below.

\section{RESULTS AND DISCUSSION}

When aiming to provide an estimation of the average number of created electron-positron pairs per incident electron emitting bremsstrahlung, we multiply the rate of 

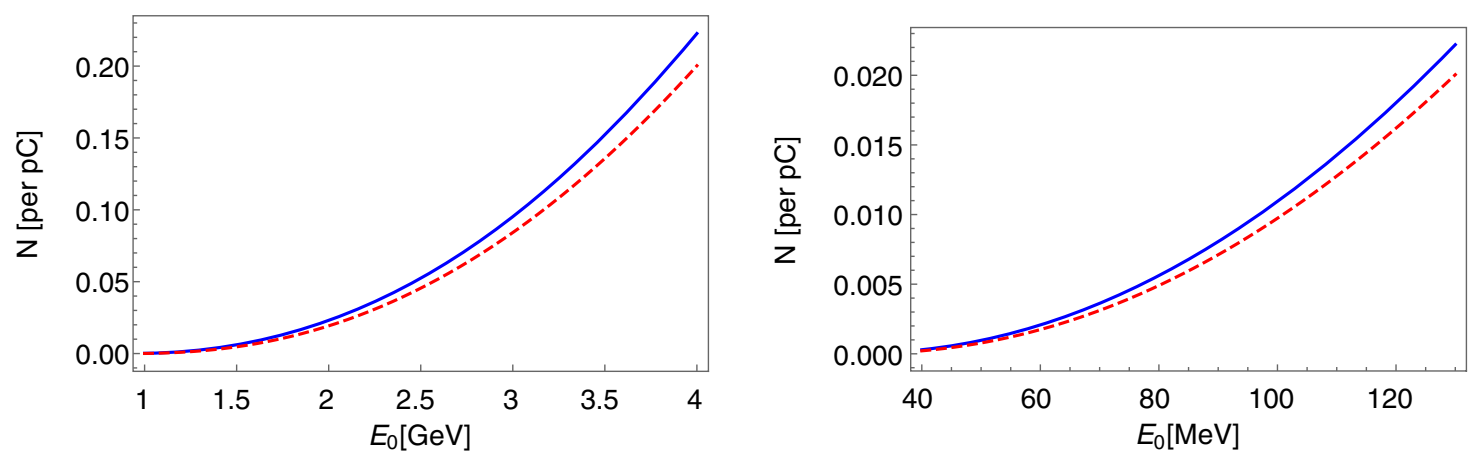

FIG. 2. Red $\left(\phi=150^{\circ}\right)$ and blue $\left(\phi=165^{\circ}\right)$ curves show the number of pairs in dependence on the initial electron energy for $\xi=0.001, \tau=100 \mathrm{fs}, w_{0}=20 \mu \mathrm{m}, L=50 \mathrm{~cm}$, and $L_{T}=7 \mathrm{~mm}$. Laser frequencies are set to $\omega=0.3 \mathrm{keV}$ for the left panel and $\omega=10 \mathrm{keV}$ for the right panel.

the process as given in Eq. (14) with the laser pulse duration and interaction volume

$$
\mathcal{N}=\tau V R_{\gamma}
$$

In the process under consideration, there is an interaction volume provided by the laser pulse which can be approximated by $V=w_{0}^{2} \pi \tau$. Moreover, the spreading beam of bremsstrahlung photons introduces a second constraint on the area, where the desirable reaction is possible. In our framework this coincides with the photon quantization volume $V_{\gamma}$ as given in Eq. (7). With the small-angle approximation it can be estimated by $\pi(\theta L)^{2} \tau$, where $\theta$ is the photon collimation angle and $L$ stands for the distance between the solid target and laser focus [see Fig. 1]. Hence, the number of pairs per incident electron reads

$$
\mathcal{N} \approx \frac{\tau w_{0}^{2}}{(\theta L)^{2}} \frac{\alpha \xi^{2} m^{2}}{4 E_{0}} \int_{f_{\min }}^{1} \frac{d f}{f} I_{\gamma}(f, l) u(s)
$$

as for an X-ray laser beam the wave length is much smaller than the beam waist $w_{0} \gg 1 / \omega$ and we perform the integration in the region where Eq. (13) provides a good approximation. Here, the normalized Mandelstam variable has been expressed as $s=\sqrt{\frac{\omega E_{0} f(1-\cos (\phi))}{2 m^{2}}}$ [compare with Eq. (7)]. Moreover, for the following discussion we introduce the usual laser field strength parameter $\xi=|e| a_{0} / m$, which serves as an indicator of whether the perturbative procedure can be applied. In our context we restrict the parameter space of $\xi$ to lie well below 1 .

Next, let us examine how the number of pairs, as given in Eq. (16), depends on the setup parameters. The rapidly evolving field of laser-wakefield electron acceleration allows for compact experimental arrangements providing electrons with energies of up to several $\mathrm{GeV}$ when using a subpetawatt-class laser [50], as provided, for example, within the HiBEF project at the European XFEL [38]. Here, we will cover electron energies in the range of $40 \mathrm{MeV}$ to $4 \mathrm{GeV}$. By penetrating through a lead target, these electrons generate a spectrum of bremsstrahlung with corresponding endpoint energies.

As the Eq. (14) represents a reliable approximation to the bremsstrahlung spectrum for restricted values of $f$, we will chose suitable laser frequencies in the domain from soft to hard $\mathrm{x}$ rays $(0.3-10 \mathrm{keV})$ in order to meet the conditions of applicability. Soft x-ray laser pulses of $0.3 \mathrm{keV}$ photon energy could be delivered, for example, by the FLASH facility at DESY in Hamburg, where photons with wavelength between $4.2-52 \mathrm{~nm}$ can be generated. Hard x-ray laser pulses with photon energies of $10 \mathrm{keV}$ and even higher are available at the European XFEL at DESY and the LCLS at Stanford [51].

For our numerical calculations, we choose the value of the laser field strength parameter as $\xi=0.001$ throughout. It corresponds to a peak intensity of $I \approx 8 \times 10^{16} \mathrm{~W} / \mathrm{cm}^{2}$ at $\omega=0.3 \mathrm{keV}$ and $I \approx 9 \times 10^{19} \mathrm{~W} / \mathrm{cm}^{2}$ at $\omega=10 \mathrm{keV}$. It is worth mentioning in this context that the photon beams from current FEL facilities are not fully coherent, even though they possess a high degree of transverse coherence and an improved longitudinal coherence as compared with synchrotron radiation sources [52]. Nevertheless, as long as the FEL intensity is kept low $(\xi \ll 1)$ and only one FEL photon participates in each reaction event-as is the case in the linear Breit-Wheeler effect- the precise photon statistics in the FEL pulse will not affect the rate of the process [53].

Figure 2 shows how the number of created positrons, which in our case coincides with the number of created pairs, depends on the energy $E_{0}$ of the incident electron beam. The left panel considers the collision of a soft x-ray laser pulse $(\omega=300 \mathrm{eV})$ with bremsstrahlung emitted from 1-4 GeV electrons. The right panel assumes, instead, that the pairs are created by an XFEL pulse of $10 \mathrm{keV}$ photon energy which collides with bremsstrahlung from $40-130 \mathrm{MeV}$ incident electrons. The red $\left(\phi=150^{\circ}\right)$ and blue $\left(\phi=165^{\circ}\right)$ curves correspond to different angles between the colliding photons (see Fig. 1). We see that, in the considered energy ranges, the number of pairs grows with increasing $E_{0}$. Even though the center-of-mass energy 


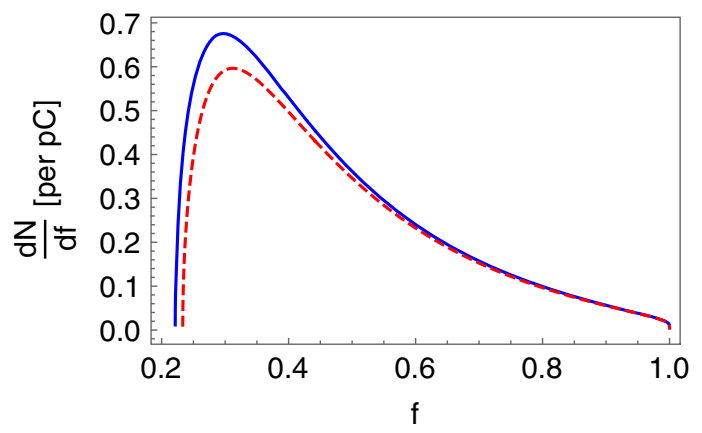

FIG. 3. Contribution of bremsstrahlung frequencies to the pair production for a lead target of thickness $L_{T}=7 \mathrm{~mm}$ and $E_{0}=4 \mathrm{GeV}, \xi=0.001, \omega=0.3 \mathrm{keV}, \tau=100 \mathrm{fs}, w_{0}=20 \mu \mathrm{m}$, $L=50 \mathrm{~cm}$ for $\phi=165^{\circ}$ (blue) and $\phi=150^{\circ}$ (red).

available for pair creation is similar in the left and right panels, respectively, the number of produced pairs is about ten times larger on the left. This outcome can be attributed to the fact that, for smaller $E_{0}$, the bremsstrahlung emission angle $\theta$ increases, which reduces the overlap with the focal region of the laser pulse and, thus, the number of bremsstrahlung photons in the interaction volume. In comparison with the aforementioned effect, the impact of the prefactor $1 / \omega^{\prime}$ in Eq. (7) is of minor importance here. We note that, for the largest electron energy considered $\left(E_{0}=4 \mathrm{GeV}\right)$ slightly more than two pairs can be generated per 10 picoCoulomb of incident electrons.

For an initial electron energy of $E_{0}=4 \mathrm{GeV}$ we examine the functional dependence of the integrand in Eq. (16) on the normalized bremsstrahlung photon energy $f$ in Fig. 3. The course of the curves reflects several tendencies. While the factor $u(s)$ in Eq. (7) always increases with growing photon energy, the Breit-Wheeler rate $R_{B W}$ itself first quickly grows from zero at the threshold, reaches a maximum at a particular value of $\omega^{\prime}$, and afterwards starts to decay-this way reflecting the influence of the factor $1 / \omega^{\prime}$ in Eq. (7). For our parameter set the value of $\omega^{\prime}$ leading to the maximum rate is approximately $1.5 \mathrm{GeV}$. The latter tendency is enhanced by the fact that the number of bremsstrahlung photons falls when their energy rises [as provided by Eq. (13)]. Due to the combination of these effects, the maximum contributions to the pair yields stem from the spectral region around $\omega^{\prime} \approx 0.3 E_{0}$. Accordingly, the electrons and positrons are created with typical energies of about $600 \mathrm{MeV}$. They are emitted predominantly in the propagation direction of the bremsstrahlung photons. In addition, we see in Fig. 3 that the maximum of the red curve for $\phi=150^{\circ}$ is slightly shifted to the right. This effect is caused by the $\phi$ dependence of the threshold energy $\sim[1-\cos (\phi)]^{-1}$. The smaller $\phi$, the larger $\omega^{\prime}$ must be to overcome it.

Further, Fig. 4 depicts the relation between the thickness of the chosen lead target $\left(Z=82, L_{\mathrm{rad}}=5.6 \mathrm{~mm}\right)$ and the expected number of created positrons. Both curves first

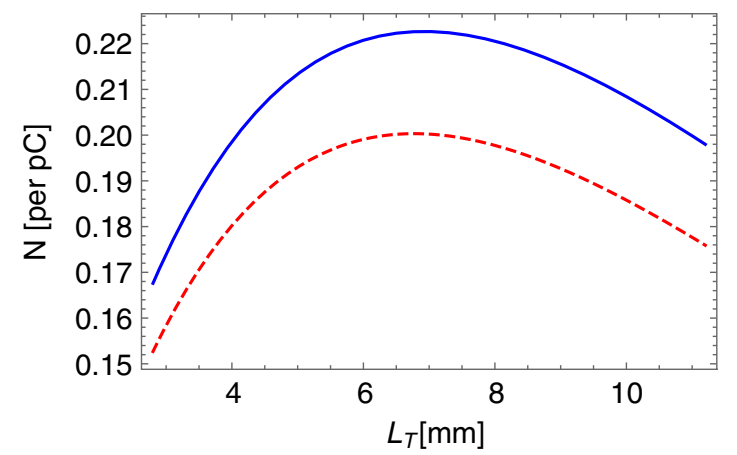

FIG. 4. Number of created pairs per $\mathrm{pC}$ for different target thicknesses $L_{T}$ of lead and $E_{0}=4 \mathrm{GeV}, \xi=0.001, \omega=0.3 \mathrm{keV}$, $\tau=100 \mathrm{fs}, w_{0}=20 \mu \mathrm{m}, L=50 \mathrm{~cm}$ for $\phi=165^{\circ}$ (blue) and $\phi=150^{\circ}$ (red).

grow with increasing target thickness, until they reach their maximum at approximately $7 \mathrm{~mm}$; from then on they decline. The maximum arises because, on the one hand, the probability for the emission of bremsstrahlung grows when the incident electrons have to travel through the target material over longer distances. On the other hand, however, emitted bremsstrahlung photons can be scattered or reabsorbed in the target; the corresponding probability increases with the target thickness as well. If the latter exceeds a certain value, the photon loss processes start to dominate over their generation, this way causing the appearance of an optimal thickness.

Finally, Fig. 5 illustrates how many pairs can be observed for the optimal parameter set $\left(E_{0}=4 \mathrm{GeV}\right.$, $L_{T}=7 \mathrm{~mm}$ ) when changing the distance between $\mathrm{x}$-ray laser focus and solid target. We see that the number of pairs to be detected decreases with growing $L$. The number scales with $L^{-2}$ as Eq. (16) shows. Similar to changes in the photon spreading angle $\theta$, we reduce the number of interacting photons when increasing the distance between the photon sources. It is interesting to note that tighter

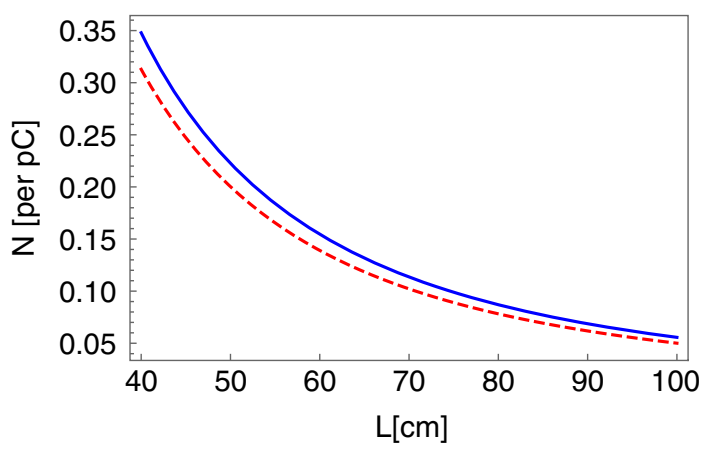

FIG. 5. Dependence of the number of created pairs per pC on the distance $L$ between solid target and laser focus. Here, the blue and red curves represent the collision angles $\phi=165^{\circ}$ and $\phi=150^{\circ}$, while the remaining parameters are set to $E_{0}=$ $4 \mathrm{GeV}, \xi=0.001, \omega=0.3 \mathrm{keV}, \tau=100 \mathrm{fs}, w_{0}=20 \mu \mathrm{m}$, and $L_{T}=7 \mathrm{~mm}$. 
focusing of the $\mathrm{x}$-ray laser does not provide a similar effect: as the number of created pairs in Eq. (16) is quadratic in both the beam waist $w_{0}$ and the parameter $\xi$, with the latter implying a linear dependence on the laser intensity, the pair yield in the linear Breit-Wheeler regime depends only on the total laser energy $E_{L}$.

Since the production of incident electron bunches of several $100 \mathrm{pC}$ up to $\approx 1 \mathrm{nC}$ is becoming feasible with LWFA [36,37], a detection of up to $\approx 350$ Breit-Wheeler pairs per shot appears approachable with experimental parameters available today or in the near future. This amount of pairs is larger than the total positron yield detected in the SLAC experiment [2,3]. It can be compared to predictions provided by similar setups involving highly energetic bremsstrahlung photons for linear and nonlinear Breit-Wheeler processes. In the linear regime of Ref. [27], up to $10^{5}$ pairs were obtained for $10^{9}$ incident electrons, corresponding to approximately $150 \mathrm{pC}$; the larger pair yield, in comparison with the present setup, may be attributed to the larger interaction volume provided by the radiation-filled hohlraum. With regard to theoretical predictions involving optical laser and bremsstrahlung photons, up to $10^{4}$ pairs per pC of $2 \mathrm{GeV}$ electrons were found in the highly nonlinear regime $(\xi=30)$ of Ref. [13]. These predicted yields assume that the detrimental impact of the bremsstrahlung beam divergence can be compensated by a suitable prefocusing of the incident electron beam. Otherwise, the number of created pairs would be reduced by several orders of magnitude, accordingly, and would reach a similar level like in the present study. In the weakly nonlinear regime $\xi \gtrsim 1$ considered in Ref. [14], on the order of 10 to $10^{6}$ pairs have been predicted per laser shot and highly energetic electron bunch of $E_{0}=$ $17.5 \mathrm{GeV}$ consisting of $6 \times 10^{9}$ particles. For the same electron beam parameters, numerical simulations in the letter of intent for the LUXE collaboration [42]when taking the envisaged experimental conditions into account-provided pair yields of $7 \times 10^{-3}$ to 350 per laser shot for $\xi$ in the range $1-7$.

\section{CONCLUSION AND OUTLOOK}

In the present paper we have theoretically examined a possible setup for detection of linear Breit-Wheeler pair creation, resulting from the interaction of an intense $\mathrm{x}$-ray laser pulse and a beam of high-energy bremsstrahlung. We have shown that by using few-GeV bremsstrahlung photons-produced from several $100 \mathrm{pC}$ of laseraccelarated incident electrons penetrating a few-mm thin high- $Z$ target-and a 100 fs soft XFEL pulse with $\sim 10^{17} \mathrm{~W} / \mathrm{cm}^{2}$ intensity, which is feasible today in the laboratory, on the order of $10^{2}-10^{3}$ positrons are expected to be created per shot. The proposed scheme thus offers a way to accomplish the long overdue experimental verification of this fundamental QED process in the linear regime, which was predicted more than 80 years ago.

\section{ACKNOWLEDGMENTS}

This work has been funded by the Deutsche Forschungsgemeinschaft (DFG) under Grant No. 416699545 within the Research Unit FOR 2783/1. H. R. acknowledges the hospitality of the Arnold Sommerfeld Center at the Ludwig-Maximilians-Universität in Munich. We thank R. W. A. Janjua for his contribution at an early stage of this study and L. Reichwein for useful discussions on LWFA.

\section{APPENDIX: FOURIER TRANSFORM OF A GAUSSIAN PULSE'S VECTOR POTENTIAL}

Let us consider the electric field component of a Gaussian pulse $\mathcal{E}=\boldsymbol{\epsilon} \mathcal{E}(x, k)$ with linear polarization $\boldsymbol{\epsilon}$ and propagation direction $z$. Within the paraxial approximation $\mathcal{E}(x, k)$ reads

$$
\mathcal{E}(x, k)=\mathcal{E}_{0} \frac{\mathrm{e}^{-\frac{(z-t)^{2}}{(\tau / 2)^{2}}}}{\sqrt{1+\zeta(z)^{2}}} \mathrm{e}^{-\frac{r^{2}}{w^{2}(z)}} \cos (\Phi(x, \omega)),
$$

where $\mathcal{E}_{0}$ is the field amplitude, $\tau$ is the pulse duration, $r^{2}=x^{2}+y^{2}$ and $w(z)=w_{0} \sqrt{1+\zeta(z)^{2}}$. Here, $w_{0}$ is the waist size of the beam, whereas $\zeta(z)=z / z_{R}$ with $z_{R}=$ $w_{0}^{2} \pi / \lambda$ denoting the Rayleigh length. Moreover, in the expression above the phase is given by

$$
\Phi(x, \omega)=\omega(z-t)+\zeta(z) \frac{r^{2}}{w^{2}(z)}-\arctan (\zeta) .
$$

The field in (A1) can be related to a vector potential $a^{\mu}=$ $\left(\varphi, \epsilon a_{0}\right)$ fulfilling the Coulomb gauge condition with $\varphi=0$. Indeed, under such restrictions $\mathcal{E}=-\boldsymbol{\epsilon} \frac{\partial a_{0}}{\partial t}$ holds. Hence, the Fourier transform of the vector potential amplitude results in

$$
\tilde{a}_{0}(\tilde{k}, k)=-\int d^{4} x \mathrm{e}^{i \tilde{k} x} \int^{t} d t^{\prime} \mathcal{E}\left(\left(t^{\prime}, \boldsymbol{x}\right), k\right) .
$$

For the sake of convenience, we first integrate over the space coordinates and subsequently perform the integrals over $t^{\prime}$ and $t$. Consequently, we obtain

$$
\begin{aligned}
\tilde{a}_{0}(\tilde{k}, k)= & -\frac{i \pi^{2} \sqrt{\pi} w_{0}^{2} \mathcal{E}_{0}}{\tilde{k}_{0}} \frac{\tau}{2} \mathrm{e}^{-\frac{\tilde{k}_{\perp}^{2} w_{0}^{2}}{4}} \\
& \times\left[\mathrm{e}^{-\frac{(\tau / 2)^{2}}{4}\left(\omega-\tilde{k}_{0}\right)^{2}} \delta\left(\tilde{k}_{z}-\tilde{k}_{0}+\frac{\tilde{k}_{\perp}^{2} w_{0}^{2}}{4 z_{R}}\right)\right. \\
& \left.+\mathrm{e}^{-\frac{(\tau / 2)^{2}}{4}\left(\omega+\tilde{k}_{0}\right)^{2}} \delta\left(\tilde{k}_{z}-\tilde{k}_{0}-\frac{\tilde{k}_{\perp}^{2} w_{0}^{2}}{4 z_{R}}\right)\right] .
\end{aligned}
$$

Notice that for long pulses, $\tau \gg 1$, the biggest contribution to the integral is provided by $\tilde{k}_{0} \approx \pm \omega$ and, hence when 
substituting $z_{R}$, the expression above can be approximated by

$$
\begin{aligned}
\tilde{a}_{0}(\tilde{k}, k)= & -\frac{i \pi \sqrt{\pi} w_{0}^{2} \mathcal{E}_{0}}{\tilde{k}_{0}} \frac{\tau}{2} \mathrm{e}^{-\frac{\tilde{z}_{\perp}^{2} w_{0}^{2}}{4}} \delta\left(\tilde{k}_{z}-\tilde{k}_{0}+\frac{\tilde{k}_{\perp}^{2}}{2 \tilde{k}_{0}}\right) \\
& \times\left[\mathrm{e}^{-\frac{(\tau / 2)^{2}}{4}\left(\omega-\tilde{k}_{0}\right)^{2}}+\mathrm{e}^{-\frac{(\tau / 2)^{2}}{4}\left(\omega+\tilde{k}_{0}\right)^{2}}\right],
\end{aligned}
$$

which coincides with the corresponding result found in [54] for $\tilde{k}_{\perp} \ll \tilde{k}_{0}$. Moreover, in the limit $\tau \rightarrow \infty$ we use an exponential representation of the Dirac delta function, $\lim _{\epsilon \rightarrow 0} \frac{1}{\sqrt{2 \pi \epsilon}} \mathrm{e}^{-\frac{x^{2}}{2 \epsilon}}=\delta(x)$, and obtain

$$
\begin{aligned}
\tilde{a}_{0}(\tilde{k}, k)= & -\frac{i 2 \pi^{3} w_{0}^{2} \mathcal{E}_{0}}{\tilde{k}_{0}} \mathrm{e}^{-\frac{\tilde{k}_{\perp}^{2} w_{0}^{2}}{4}} \delta\left(\tilde{k}_{z}-\tilde{k}_{0}+\frac{\tilde{k}_{\perp}^{2}}{2 \tilde{k}_{0}}\right) \\
& \times\left(\delta\left(\omega-\tilde{k}_{0}\right)+\delta\left(\omega+\tilde{k}_{0}\right)\right),
\end{aligned}
$$

which corresponds to the vector potential of a Gaussian beam.
[1] G. Breit and J. A. Wheeler, Collision of two light quanta, Phys. Rev. 46, 1087 (1934).

[2] D. L. Burke et al., Positron Production in Multiphoton Light-by-Light Scattering, Phys. Rev. Lett. 79, 1626 (1997).

[3] C. Bamber et al., Studies of nonlinear QED in collisions of 44.6 GeV electrons with intense laser pulses, Phys. Rev. D 60, 092004 (1999).

[4] V. I. Ritus, Quantum effects of the interaction of elementary particles with an intense electromagnetic field, J. Sov. Laser Res. 6, 497 (1985).

[5] H. R. Reiss, Special analytical properties of ultrastrong coherent fields, Eur. Phys. J. D 55, 365 (2009).

[6] H. Hu, C. Müller, and C. H. Keitel, Complete QED Theory of Multiphoton Trident Pair Production in Strong Laser Fields, Phys. Rev. Lett. 105, 080401 (2010).

[7] F. Ehlotzky, K. Krajewska, and J. Z. Kamiński, Fundamental processes of quantum electrodynamics in laser fields of relativistic power, Rep. Prog. Phys. 72, 046401 (2009).

[8] A. Di Piazza, C. Müller, K. Z. Hatsagortsyan, and C. H. Keitel, Extremely high-intensity laser interactions with fundamental quantum systems, Rev. Mod. Phys. 84, 1177 (2012).

[9] K. Krajewska and J. Z. Kamiński, Breit-Wheeler process in intense short laser pulses, Phys. Rev. A 86, 052104 (2012).

[10] K. Krajewska and J.Z. Kamiński, Coherent combs of antimatter from non-linear electron-positron-pair creation, Phys. Rev. A 90, 052108 (2014).

[11] A. Di Piazza, Nonlinear Breit-Wheeler Pair Production in a Tightly Focused Laser Beam, Phys. Rev. Lett. 117, 213201 (2016).

[12] S. Meuren, C. H. Keitel, and A. Di Piazza, Semiclassical picture for electron-positron photoproduction in strong laser fields, Phys. Rev. D 93, 085028 (2016).

[13] T. G. Blackburn and M. Marklund, Nonlinear Breit-Wheeler pair creation with bremsstrahlung $\gamma$ rays, Plasma Phys. Controlled Fusion 60, 054009 (2018).

[14] A. Hartin, A. Ringwald, and N. Tapia, Measuring the boiling point of the vacuum of quantum electrodynamics, Phys. Rev. D 99, 036008 (2019).

[15] S. Villalba-Chávez and C. Müller, Photo-production of scalar particles in the field of a circularly polarized laser beam, Phys. Lett. B 718, 992 (2013).
[16] M. J. A. Jansen and C. Müller, Strongly enhanced pair production in combined high- and low-frequency laser fields, Phys. Rev. A 88, 052125 (2013).

[17] T. Heinzl, A. Ilderton, and M. Marklund, Finite size effects in stimulated laser pair production, Phys. Lett. B 692, 250 (2010).

[18] A. I. Titov, H. Takabe, B. Kämpfer, and A. Hosaka, Enhanced Subthreshold e+e- Production in Short Laser Pulses, Phys. Rev. Lett. 108, 240406 (2012).

[19] A. I. Titov, B. Kämpfer, A. Hosaka, T. Nousch, and D. Seipt, Determination of the carrier envelope phase for short, circularly polarized laser pulses, Phys. Rev. D 93, 045010 (2016).

[20] M. J. A. Jansen, J.Z. Kamiński, K. Krajewska, and C. Müller, Strong-field Breit-Wheeler pair production in short laser pulses: Relevance of spin effects, Phys. Rev. D 94, 013010 (2016).

[21] M. J. A. Jansen and C. Müller, Strong-field Breit-Wheeler pair production in two consecutive laser pulses with variable time delay, Phys. Lett. B 766, 71 (2017).

[22] Q. Z. Lv, S. Dong, Y. T. Li, Z. M. Sheng, Q. Su, and R. Grobe, Role of the spatial inhomogeneity on the laserinduced vacuum decay, Phys. Rev. A 97, 022515 (2018).

[23] A. I. Titov, H. Takabe, and B. Kämpfer, Breit-Wheeler process in short laser double pulses, Phys. Rev. D 98, 036022 (2018).

[24] Y.-Y. Chen, P.-L. He, R. Shaisultanov, K. Z. Hatsagortsyan, and C. H. Keitel, Polarized Positron Beams via Intense Two-Color Laser Pulses, Phys. Rev. Lett. 123, 174801 (2019).

[25] The term 'linear' refers to the linear dependence of the pair production rate on the applied photon beam intensity.

[26] B. King, H. Gies, and A. Di Piazza, Pair production in a plane wave by thermal background photons, Phys. Rev. D 86, 125007 (2012).

[27] O. J. Pike, F. Mackenroth, E. G. Hill, and S. J. Rose, A photon-photon collider in a vacuum hohlraum, Nat. Photonics 8, 434 (2014).

[28] I. Drebot, D. Micieli, E. Milotti, V. Petrillo, E. Tassi, and L. Serafini, Matter from light-light scattering via BreitWheeler events produced by two interacting Coulomb sources, Phys. Rev. Accel. Beams 20, 043402 (2017). 
[29] X. Ribeyre, E. d'Humières, O. Jansen, S. Jequier, V. T. Tikhonchuk, and M. Lobet, Pair creation in collision of $\gamma$-ray beams produced with high-intensity lasers, Phys. Rev. E 93, 013201 (2016).

[30] I. J. Yu, H. Y. Lu, T. Takahashi, R. H. Hu, Z. Gong, W. J. Ma, Y. S. Huang, C. E. Chen, and X. Q. Yan, Creation of Electron-Positron Pairs in Photon-Photon Collisions Driven by 10-PW Laser Pulses, Phys. Rev. Lett. 122, 014802 (2019); T. Wang and A. Arefiev, Comment on Creation of Electron-Positron Pairs in Photon-Photon Collisions Driven by 10-PW Laser Pulses, Phys. Rev. Lett. 125, 079501 (2020).

[31] T. Wang, X. Ribeyre, Z. Gong, O. Jansen, E. d'Humières, D. Stutman, T. Toncian, and A. Arefiev, Power scaling for collimated $\gamma$-ray beams generated by structured laserirradiated targets and its application to two-photon pair production, Phys. Rev. Applied 13, 054024 (2020).

[32] A. Golub, R. Egger, C. Müller, and S. Villalba-Chávez, Dimensionality-Driven Photoproduction of Massive Dirac Pairs near Threshold in Gapped Graphene Monolayers, Phys. Rev. Lett. 124, 110403 (2020).

[33] T. Piran, The physics of gamma-ray bursts, Rev. Mod. Phys. 76, 1143 (2005).

[34] R. Ruffini, V. G. Vereshchagin, and S. S. Xue, Electronpositron pairs in physics and astrophysics: From heavy nuclei to black holes, Phys. Rep. 487, 1 (2010).

[35] It is interesting to note that (quasireal) photon-photon reactions can also be studied in high-energy proton-proton collisions at the Large Hadron Collider: see, e.g., A. Abulencia et al. (CDF Collaboration), Observation of Exclusive Electron-Positron Production in Hadron-Hadron Collisions, Phys. Rev. Lett. 98, 112001 (2007); J. de Favereau de Jeneret et al., High energy photon interactions at the LHC, arXiv:0908.2020; G. Aad et al. (ATLAS Collaboration), Observation of photon-induced $W^{+} W^{-}$ production in $p p$ collisions at $\sqrt{s}=13 \mathrm{TeV}$ using the ATLAS detector, arXiv:2010.04019.

[36] A. J. Gonsalves et al., Petawatt Laser Guiding and Electron Beam Acceleration to $8 \mathrm{GeV}$ in a Laser-Heated Capillary Discharge Waveguide, Phys. Rev. Lett. 122, 084801 (2019).

[37] G. Götzfried, A. Döpp, M. F. Gilljohann, F. M. Foerster, H. Ding, S. Schindler, G. Schilling, A. Buck, L. Veisz, and S. Karsch, Physics of Nanocoulomb-Class Electron Beams in Laser-Plasma Wakefields, Phys. Rev. X 10, 041015 (2020).

[38] http://www.hibef.eu/.

[39] H. Chen, S. C. Wilks, J. D. Bonlie, E. P. Liang, J. Myatt, D. F. Price, D. D. Meyerhofer, and P. Beiersdorfer, Relativistic Positron Creation Using Ultraintense Short Pulse Lasers, Phys. Rev. Lett. 102, 105001 (2009).

[40] G. Sarri et al., Table-Top Laser-Based Source of Femtosecond, Collimated, Ultrarelativistic Positron Beams, Phys. Rev. Lett. 110, 255002 (2013).
[41] H. R. Reiss, Production of Electron Pairs from a Zero-Mass State, Phys. Rev. Lett. 26, 1072 (1971).

[42] H. Abramowicz et al., Letter of intent for the LUXE experiment, arXiv:1909.00860.

[43] W. Greiner and J. Reinhardt, Quantum Electrodynamics (Springer-Verlag, Berlin, Heidelberg, 2003).

[44] A. I. Akhiezer and V. B. Berestetskii, Quantum Electrodynamics, 2nd ed. (Wiley, New York, 1965).

[45] Depletion effects of the laser field can safely be neglected in the present situation; for corresponding studies, we refer to J. Bergou and S. Varro, Nonlinear scattering processes in the presence of a quantised radiation field: II. Relativistic treatment, J. Phys. A 14, 2281 (1981); D. Seipt, T. Heinzl, M. Marklund, and S. S. Bulanov, Depletion of Intense Fields, Phys. Rev. Lett. 118, 154803 (2017); A. Ilderton and D. Seipt, Backreaction on background fields: A coherent state approach, Phys. Rev. D 97, 016007 (2018).

[46] E. S. Fradkin, D. M. Gitman, and S. V. Shvartsman, Quantum Electrodynamics with Unstable Vacuum (SpringerVerlag, Berlin, Heidelberg. 1961).

[47] A. Blinne, H. Gies, F. Karbstein, C. Kohlfürst, and M. Zepf, Photon-photon scattering at the high-intensity frontier: Paraxial beams, J. Phys. Conf. Ser. 1206, 012016 (2019).

[48] M. Stearns, Mean square angles of bremsstrahlung and pair production, Phys. Rev. 76, 836 (1949).

[49] Y.-S. Tsai, Pair production and bremsstrahlung of charged leptons, Rev. Mod. Phys. 46, 815 (1974).

[50] W. P. Leemans et al., Multi-GeV Electron Beams from Capillary-Discharge-Guided Subpetawatt Laser Pulses in the Self-Trapping Regime, Phys. Rev. Lett. 113, 245002 (2014).

[51] https://lcls.slac.stanford.edu/parameters.

[52] G. Geloni, E. Saldin, L. Samoylova, E. Schneidmiller, H. Sinn, T. Tschentscher, and M. Yurkov, Coherence properties of the European XFEL, New J. Phys. 12, 035021 (2010); E. A. Schneidmiller and M. V. Yurkov, Coherence properties of the radiation from FLASH, J. Mod. Opt. 63, 293 (2016); C. Gutt et al., Single Shot Spatial and Temporal Coherence Properties of the SLAC Linac Coherent Light Source in the Hard X-Ray Regime, Phys. Rev. Lett. 108, 024801 (2012).

[53] The impact of the photon statistics was rigorously studied in the context of multiphoton ionization processes. In particular, it was shown that the probability for one-photon absorption is independent of the statistical properties of the light beam: see, e.g., S. Carusotto, G. Fornaca, and E. Polacco, Two-photon absorption and coherence, Phys. Rev. 157, 1207 (1967); H. D. Simaan and R. Loudon, Quantum statistics of single beam two-photon absorption, J. Phys. A 8, 539 (1975); R. Loudon, The Quantum Theory of Light (Oxford University Press, Oxford, 2001).

[54] W. J. Waters and B. King, On beam models and their paraxial approximation, Laser Phys. 28, 015003 (2018). 